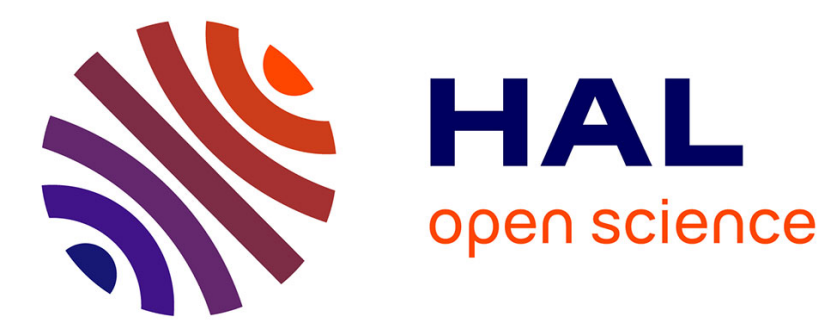

\title{
A local approach to reasoning under inconsistency in stratified knowledge bases
}

\author{
Salem Benferhat, Didier Dubois, Henri Prade
}

\section{To cite this version:}

Salem Benferhat, Didier Dubois, Henri Prade. A local approach to reasoning under inconsistency in stratified knowledge bases. 3rd European conference Symbolic and Quantitative Approaches to Reasoning and Uncertainty (ESCQARU 1995), Jul 1995, Fribourg, Switzerland. pp.36-43, 10.1007/3540-60112-0_5. hal-03299846

\section{HAL Id: hal-03299846 https://hal-univ-artois.archives-ouvertes.fr/hal-03299846}

Submitted on 3 Sep 2021

HAL is a multi-disciplinary open access archive for the deposit and dissemination of scientific research documents, whether they are published or not. The documents may come from teaching and research institutions in France or abroad, or from public or private research centers.
L'archive ouverte pluridisciplinaire HAL, est destinée au dépôt et à la diffusion de documents scientifiques de niveau recherche, publiés ou non, émanant des établissements d'enseignement et de recherche français ou étrangers, des laboratoires publics ou privés. 


\title{
A local approach to reasoning under inconsistency in stratified knowledge bases
}

\author{
Salem Benferhat, Didier Dubois and Henri Prade \\ I.R.I.T. - C.N.R.S. - Univ. Paul Sabatier - 31062 Toulouse Cedex, France \\ e-mail: \{benferha, dubois, prade\}@irit.irit.fr
}

\begin{abstract}
This paper investigates an approach for reasoning under inconsistency in a "local" way, in prioritized knowledge bases. In such bases, the higher the layer, the more certain, the more reliable are the formulas stored in this layer. The proposed approach is based on the notion of (consistent) argument whose strength depends on the layer of the least certain formulas involved in the argument. Each formula in the base is also associated with a "level of paraconsistency" which reflect to what extent there exists arguments that support both a formula and its negation. Three consequence relations are presented and compared. Two of them aim at maximizing the certainty degree and/or at minimizing the level of paraconsistency of the conclusion. The third one produces consequences that are safely supported in the sense that there exists an undefeated argument for them (whose certainty is greater than its paraconsistency).
\end{abstract}

\section{Introduction}

Inconsistency may appear when a plausible consequence, obtained under incomplete information, has to be revised because further information is available. This issue has been extensively investigated in the nonmonotonic reasoning literature. In this paper we rather view inconsistency as being caused by the use (and the fusion) of multiple sources of information. Even if each source $i$ of information provides a consistent knowledge base $\mathrm{K}_{\mathrm{i}}$, it may often happen in practice that the result of concatenating the $\mathrm{K}_{\mathrm{i}}$ will be inconsistent. In such a situation, we further assume that the knowledge bases $\mathrm{K}_{\mathrm{i}}$ store "regular" pieces of information rather than default rules liable to have explicit exceptions. In this paper, we will only deal with one inconsistent and prioritized (or if we prefer, stratified) knowledge base, denoted by $\Sigma$, which can be seen as the result of putting together several consistent knowledge bases. In this context, the introduction of priorities between pieces of information in $\Sigma$ can be explained by the two following scenarios:

- Each consistent knowledge base $\mathrm{K}_{\mathrm{i}}$, issued from a source of information, is "flat" (i.e., without any priority between their elements). But there is a total pre-ordering between the sources of information according to their reliability. In this case merging different sources of information leads to a prioritized knowledge base $\sum$, where the certainty level of each formula reflects the reliability of the (best) source which provides it. A particular case is when each piece of information in $\Sigma$ is supported by a different source.

- All sources of information are equally reliable (and thus have the same level of reliability), but inside each consistent knowledge base $\mathrm{K}_{\mathrm{i}}$ there exists a preference relation between pieces of information given by an expert, which rank-orders them according to their level of certainty. Here again, the combination of the different sources of information gives an uncertain knowledge base, provided that the scales of uncertainty used in each knowledge base $\mathrm{K}_{\mathrm{i}}$ are commensurate.

In this paper, we only consider a finite propositional language denoted by $\mathscr{L}$. The symbol $\vdash$ represents the classical consequence relation, Greek letters $\alpha, \beta, \delta, \ldots, \phi$, 
$\psi, \chi, \ldots$ represent formulas. Let $\Sigma$ be a set of propositional formulas, possibly inconsistent but not deductively closed. This paper deals with layered knowledge bases of the form $\sum=\mathrm{S}_{1} \cup \ldots \cup \mathrm{S}_{\mathrm{n}}$.

Possibilistic logic (e.g., [6]) offers a way of deriving non-trivial conclusions from an inconsistent knowledge base by taking advantage of the stratification of the base $\Sigma=\mathrm{S}_{1} \cup \ldots \cup \mathrm{S}_{\mathrm{n}}$ where formulas in $\mathrm{S}_{\mathrm{i}}$ are considered as strictly more certain than the ones in $S_{j}$ if $j>i$. This stratification is modelled in possibilistic logic [6] by attaching a weight $a \in[0,1]$ to each formula with the convention that $\left(\phi \mathrm{a}_{\mathrm{i}}\right) \in \mathrm{S}_{\mathrm{i}}, \forall \mathrm{i}$ and $\mathrm{a}_{1}=1>$ $a_{2}>\ldots>a_{n}>0$; for instance take $a_{j}=1 / j$. From now on, we will use a possibilistic representation of prioritized knowledge bases. A level of (partial) inconsistency for the base is computed as the level $a_{j+1}$ such that $S_{1} \cup \ldots \cup S_{j}$ is consistent but $S_{1} \cup \ldots$ $\cup S_{j+1}$ is not (consistency of $\sum$ corresponds to inconsistency of level 0 ). The conclusions derived from $S_{1} \cup \ldots \cup S_{i}$ with $a_{i}$ strictly greater than the level of inconsistency are considered as well-grounded, since they are above the inconsistency level of $\sum$ where inconsistency occurs, and more precisely since they can be logically deduced (in a non trivial way) using the most reliable part of $\sum$ only. Although possibilistic reasoning can be successfully used for handling default information [1], it has two limitations for reasoning under inconsistency. Namely, formulas which do not take part to the inconsistency of the base but which have a level of certainty smaller or equal to the level of inconsistency of the base are not used in the inference process. Moreover, the user receives no information on the levels of certainty (smaller or equal to the level of inconsistency) of the formulas (if any) which contradict some of the formulas used for deriving the conclusion under consideration. The approach presented in this paper remedies these limitations. More precisely, the paper investigates three consequence relations capable of inferring non-trivial conclusions from an inconsistent knowledge base. In each case a level of paraconsistency is computed for each conclusion, which assesses to what extent there exists formulas which are somewhat certain in the base and which contradict some of the formulas used in the proof of the conclusion. These consequence relations treat inconsistency in a local way, by contrast with most of the approaches developed in the literature which work in a global way. See, e.g., Rescher \& Manor[7]; Brewka[4]; Benferhat et al.[2].

The paper is organized as follows. Section 2 gives the background needed for the reading of this paper. Section 3 introduces the three proposed inconsistency-tolerant consequence relations which treat inconsistency in a local way. A comparative study between these consequence relations is given in Section 4.

\section{Background}

Throughout this paper, we denote sub-bases by capital letters A,B,C... and they are also represented in a stratified way. For the sake of simplicity, we will use the notation $\mathrm{A}-\psi$ to denote that $\psi$ is a logical consequence of the formulas of $\mathrm{A}$ when we forget their weights. A sub-base A of $\sum$ is said to be consistent if it is not possible to deduce a contradiction from A, i.e., $A \nvdash \_\perp$.

Def. 1: A sub-base A of $\sum$ is said to be minimal inconsistent if and only if it satisfies the two following requirements: i) $\mathrm{A} \leftarrow \perp$, and ii) $\forall(\phi$ a $) \in \mathrm{A}, \mathrm{A}-\{(\phi \mathrm{a})\} \mathcal{F}^{\circ} \perp$.

From now on, we denote by $\operatorname{Inc}(\Sigma)$ the set of formulas belonging to at least one minimal inconsistent sub-base of $\sum$, namely:

$\operatorname{Inc}\left(\sum\right)=\left\{(\phi \mathrm{a}), \exists \mathrm{A} \subseteq \sum\right.$, such that $(\phi \mathrm{a}) \in \mathrm{A}$ and $\mathrm{A}$ is minimal inconsistent $\}$. 
When we remove from $\Sigma$ all elements of $\operatorname{Inc}\left(\sum\right)$, the resulting base is called the free base of $\sum$, denoted by Free $(\Sigma)[1]$. In other words, the set Free $\left(\sum\right)$ contains all the formulae, called free formulas, which are not involved in any inconsistency of the knowledge base $\sum$. Clearly Free $(\Sigma)$ may be empty; $\operatorname{Inc}(\Sigma)=\varnothing$ when $\Sigma$ is consistent. We now give the notion of free-consequence:

Def. 2: A formula $\phi$ is said to be a free consequence of $\Sigma$, denoted by $\Sigma-$ Free $\phi$, if and only if $\phi$ is logically entailed from $\operatorname{Free}(\Sigma)$, namely: $\Sigma \models$ Free $\phi$ iff $\operatorname{Free}(\Sigma) \vdash \phi$.

It is not hard to see that the Free-inference relation is very conservative, since it corresponds to a maximal revision of $\sum$, deleting all formulas involved in a conflict. We finish this section by defining the notion of argument:

Def. 3: A consistent sub-base A of $\sum$ is said to be an argument to a degree $a$ for a formula $\phi$ if it satisfies the following conditions: (i) $\mathrm{A}-\phi$, (ii) $\forall(\psi \mathrm{b}) \in \mathrm{A}$, $\mathrm{A}-\{(\psi \mathrm{b})\} \leftarrow \mathcal{K} \phi$, and (iii) $\mathrm{a}=\min \left\{\mathrm{a}_{\mathrm{i}} /\left(\phi_{\mathrm{i}} \mathrm{a}_{\mathrm{i}}\right) \in \mathrm{A}\right\}$.

An argument for $\phi$ is then a minimal consistent sub-base of $\sum$ which entails logically $\phi$ (in the sense of possibilistic logic, which requires condition (iii)). Note that this notion of argument is an extension of the one proposed by Simari and Loui [8]. These authors apply the notion of arguments to default reasoning (arguments are used to determine the relation of specificity between pieces of default information).

\section{Local inconsistency-tolerant consequence relations}

This section presents an approach to deal with inconsistent prioritized knowledge bases in a "local" way and studies three consequence relations in this framework. As in possibilistic logic, levels of priority or of certainty attached to formulas are used to distinguish between strong and less strong arguments in favour of a proposition or of its contrary. However it is possible to go one step further in the use of the certainty or priority levels by i) attaching to each proposition $\phi$ in the knowledge base not only its certainty weight $a$ (obtained by computing the strongest argument in favour of $\phi$ in the sense of possibilistic logic), but also the weight $b$ attached to the strongest argument in favour of $\neg \phi$ if any, and by ii) inferring from weighted premises such as $(\phi \mathrm{a}$ ) by propagating the weights $a$ and $b$. It will enable us to distinguish between consequences obtained only from "free" propositions in the knowledge base $\sum$ for which $b=0$ (i.e., propositions for which there is no argument in $\sum$ in favour of their negation), and consequences obtained using also propositions which are not free (for which there exists also a weighted argument in favour of their negation even if the latter has a smaller weight) ${ }^{1}$.

More formally, the idea is first to attach to any formula in the stratified knowledge base $\sum$ two numbers $a$ and $b$ reflecting respectively the extent to which we have some certainty that the formula is true and the extent to which we have some certainty that the formula is false. When $b=0$ then $\phi$ is free since $\phi$ is not involved in the inconsistency of $\sum$ (otherwise there would exist an argument in favour of $\neg \phi$ ). When $a \neq 0$ and $b \neq 0, \phi$ is said to be paraconsistent. In the general case, we shall say that the pair $(\phi \neg \phi)$ has a level of "paraconsistency" equal to min(a,b). Classically and roughly speaking, the idea of paraconsistency is to say that $\phi$ is paraconsistent if there is a reason for stating both $\phi$ and $\neg \phi$. It corresponds to the situation where we have conflicting information about $\phi$. This is why we speak here of paraconsistent

1 [5] already includes a brief suggestion of this approach by proposing an extension of the possibilistic resolution principle, handling paraconsistency degrees. 
information when $\min (a, b)>0$, although the approaches presented in the following depart from classical paraconsistent logics (see [3] for their extension of these logics to the possibilistic framework). From now on, we denote by $\sum^{\prime}$ the set of triples ( $\phi$ a b) such that $\left(\phi \mathrm{a}_{\mathrm{i}}\right)$ belongs to $\sum, a$ is the weight attached to the strongest ${ }^{2}$ argument in favour of $\phi$ and $b$ is the weight attached to the strongest ${ }^{2}$ argument in favour of its contrary $\neg \phi$. When $\mathrm{b} \geq \mathrm{a}$ then $\phi$ is said to be defeated by some argument. In the other case $(\mathrm{a}>\mathrm{b}) \phi$ is said to be an argumentative consequence of $\sum[2]$.

To see if a formula $\psi$ is a plausible consequence of $\sum$, we first check if there is an argument in favour of $\psi$ in $\sum$. It is clear that if there is no argument in favour of $\psi$ in $\sum$ then $\psi$ cannot be a plausible consequence of $\sum$, since we have no reason to believe $\psi$ in $\sum$. Assume that we have an argument $\mathrm{A}$ in favour of $\psi$ where all elements of $\mathrm{A}$ are free formulas, then $\psi$ can be considered as a plausible consequence of $\sum$ (i.e., $\psi$ is a free consequence of $\Sigma$ ). The situation differs if some elements of $\mathrm{A}$ are not free, and here we must be more careful in our inference. Indeed, let $\phi$ be a formula in A (the argument for $\psi$ ) such that there exists an argument in $\sum$ which supports $\neg \phi$ with a certainty degree $\mathrm{b}$ higher than the one, $a$, of $\phi$ (namely $\phi$ is defeated), then the conclusion $\psi$ must not be longer considered as a plausible consequence of $\sum$, as soon as, due to $\mathrm{a}<\mathrm{b}$, we do not consider $\phi$ as a plausible consequence of $\sum$ (although ( $\phi \mathrm{a} \mathrm{b}$ ) is in $\left.\sum^{\prime}\right)$.

Once $\Sigma^{\prime}$ is constructed, we are going to associate two degrees to a conclusion $\psi$ derived from an argument $\mathrm{A}$ in $\sum$ : $\operatorname{Cert}(\mathrm{A})$, called the certainty degree of $\psi$ using $\mathrm{A}$, which just evaluates to what extent $\psi$ is supported by an argument A, and Para(A), called the paraconsistency degree of $\psi$ using A, which evaluates our degree of doubt to conclude $\psi$ using A. These two measures are computed in the following way:

$$
\begin{aligned}
& \operatorname{Cert}(A)=\min \left\{a_{i} \mid\left(\phi_{i} a_{i} b_{i}\right) \in \Sigma^{\prime} \text { and }\left(\phi_{i} a\right) \in A\right\}, \\
& \operatorname{Para}(A)=\max \left\{b_{i} \mid\left(\phi_{i} a_{i} b_{i}\right) \in \Sigma^{\prime} \text { and }\left(\phi_{i} a\right) \in A\right\} .
\end{aligned}
$$

Cert(A) estimates the strength of argument A, and Para(A) its brittleness. In general, we can have several arguments which support $\psi$ and we denote the set of all pairs thus obtained for $\psi$ by:

$$
\operatorname{Label}(\psi)=\left\{\left(\operatorname{Cert}\left(\mathrm{A}_{\mathrm{i}}\right), \operatorname{Para}\left(\mathrm{A}_{\mathrm{i}}\right)\right) \mid \mathrm{A}_{\mathrm{i}} \text { is an argument for } \psi\right\}
$$

From Label $(\psi)$, we may think of two criteria to select the best argument for $\psi$ : the certainty degree and the paraconsistency degree induced by the argument. It is clear that the best argument for $\psi$ is the one which allows to deduce $\psi$ with the highest certainty degree and the lowest paraconsistency degree. But in general, such argument does not always exist. Namely, we can have an argument for $\psi$ with a high certainty degree, but also with a high paraconsistency degree, and another for $\psi$ with low certainty and low paraconsistency. Then it is less obvious how to choose the best argument. We may: first either minimize the paraconsistency degree of a conclusion, or first maximize the certainty degree. These two possibilities lead to two definitions of inconsistency-tolerant consequence relations that we examine now.

Minimizing paraconsistency: One way to select the best argument among those which support $\psi$ is to give a preference to arguments which minimize the paraconsistency degree of $\psi$. The knowledge base $\sum$ can be viewed as decomposed into two sub-parts: the consistent (or free) sub-part, represented by $\operatorname{Free}(\Sigma)$, and the paraconsistent sub-part, represented by $\operatorname{Inc}(\Sigma)$. In this approach, the best argument for $\psi$ is the one (if it exists) obtained from $\operatorname{Free}(\Sigma)$ even if the certainty degree of $\psi$ induced by this argument is very low. If such argument does not exist in $\operatorname{Free}(\Sigma)$, then

2 If there is an argument in favour of $\phi($ resp. $\neg \phi)$ to a degree $c$ (resp. d) then $a \geq c$ (resp. b $\geq d$ ). 
the best argument for $\psi$ is obtained by i) using formulas from $\operatorname{Free}(\Sigma)$ as much as possible, ii) when necessary, using formulas from $\operatorname{Inc}\left(\sum\right)$ with the lowest paraconsistency degrees. More formally:

Def. 4: Let LabelPara $(\psi)$ be the subset of Label $(\psi)$ obtained by choosing the pairs with the lowest paraconsistency value. Let $(\operatorname{Cert}(A), \operatorname{Para}(A))$ be a pair of LabelPara $(\psi)$ such that Cert(A) has the highest certainty value. Then $\psi$ is said to be $P C$-consequence of $\sum$ (PC: short for "first paraconsistency then certainty"), denoted by $\Sigma-\mathrm{PC} \psi$, iff $\operatorname{Cert}(\mathrm{A})>\operatorname{Para}(\mathrm{A})$.

Maximizing certainty: There is another view to selecting the best argument for $\psi$, where we prefer the argument which maximizes the certainty of $\psi$. This approach agrees with the principle that the lower is the certainty degree of formulas in a given argument, the lower is our degree of acceptation of the conclusion given by this argument.

Def. 5: Let LabelCert $(\psi)$ be the subset of Label $(\psi)$ obtained by choosing pairs with the highest certainty value. Let $(\operatorname{Cert}(\mathrm{A}), \operatorname{Para}(\mathrm{A}))$ be a pair in LabelCert $(\psi)$ such that $\operatorname{Para}(\mathrm{A})$ has the lowest paraconsistency degree. Then $\psi$ is said to be a $C P$-consequence of $\sum$ (CP: short for "first certainty then paraconsistency"), denoted by $\Sigma-\mathrm{CP} \psi$, iff $\operatorname{Cert}(\mathrm{A})>\operatorname{Para}(\mathrm{A})$.

Definition 5 can be explained in a simpler way: to check if a conclusion $\psi$ is a CPconsequence of $\sum$, first compute the greatest weight attached to the strongest argument, say A, for $\psi$. Next, compute the paraconsistency degree b of $\psi$ with respect to A. Finally, if $\mathrm{a}>\mathrm{b}$ then conclude that $\psi$ is a $\mathrm{CP}$-consequence of $\sum$. If the strongest argument $\mathrm{A}$ is not unique, take the one with the smallest paraconsistency degree.

Proceeding level by level: In this sub-section, we present a third local inference relation. We suggest that a formula $\psi$ is a plausible consequence of $\sum$, if there exists a degree $a$ such that $\psi$ is a free-consequence of a sub-base of $\sum$ composed of all formulas of $\sum$ having a certainty degree higher or equal to $a$. It means that all formulas which are involved in the entailment of $\psi$ must be either free formulas or have a level of paraconsistency less than $a$ and thus less than the degree of certainty of $\psi$. More formally, let $\sum_{\mathrm{a}}=\left\{(\phi \mathrm{b}) \mid(\phi \mathrm{b}) \in \sum\right.$ and $\left.\mathrm{b} \geq \mathrm{a}\right\}$ be the set of formulas of $\sum$ having a certainty degree higher or equal to $a$, and $\operatorname{Free}\left(\Sigma_{\mathrm{a}}\right)$ denotes the set of free formulas in $\Sigma_{\mathrm{a}}$. It is clear that $\operatorname{Free}\left(\sum_{\mathrm{a}}\right)$ is different from $(\operatorname{Free}(\Sigma))_{\mathrm{a}}$, and more precisely we have the following relation: $(\operatorname{Free}(\Sigma))_{\mathrm{a}} \subseteq \operatorname{Free}\left(\Sigma_{\mathrm{a}}\right)$. Note also that there is no inclusion relation between Free $\left(\Sigma_{\mathrm{a}}\right)$ and $\operatorname{Free}\left(\Sigma_{\mathrm{b}}\right)$ where $\mathrm{a}>\mathrm{b}$. Indeed, since $\Sigma_{\mathrm{a}} \subset \Sigma_{\mathrm{b}}, \Sigma_{\mathrm{b}}$ may include new free formulas but also some which contradict formulas which were free in $\Sigma_{\mathrm{a}}$. We define the inference relation that generates plausible results of $\sum$ :

Def. 6: A formula $\psi$ is said to be a safely supported consequence of $\sum$, denoted by $\Sigma-\mathrm{SS} \psi$, if and only if there exists a positive number $a$ such that $\Sigma_{\mathrm{a}} \vdash$ Free $\psi$.

The degree of certainty of $\psi$ is the greatest number $a$ such that Free $\left(\Sigma_{\mathrm{a}}\right)-\psi$, namely

$$
\operatorname{Cert}(\psi)=\max \left\{\mathrm{a}, \Sigma_{\mathrm{a}} \vdash \text { Free } \psi\right\} \text {. }
$$

Thus, $\forall \mathrm{b}>\operatorname{Cert}(\psi), \Sigma_{\mathrm{b}} \nvdash^{*}$ Free $\psi$. The paraconsistency degree of $\psi$ is the lowest paraconsistency degree induced by arguments supporting $\psi$ in $\sum_{\operatorname{Cert}}(\psi)$, namely:

$$
\operatorname{Para}(\psi)=\min \left\{\operatorname{Para}(\mathrm{A}) / \mathrm{A} \subseteq \sum_{\operatorname{Cert}}(\psi) \text { and } \mathrm{A} \text { is an argument for } \psi\right\} \text {. }
$$

Notice that if for a given degree $\mathrm{c}<\operatorname{Cert}(\psi)$ we have $\Sigma_{\mathrm{c}} \nvdash^{\leftarrow}$ Free $\psi$, then there is no longer a proof of $\psi$ in $\Sigma_{\mathrm{c}}$ made only of free formulas and that at least one of the 
formulas, say $\phi$, used in the free proof of $\psi$ from $\Sigma_{\mathrm{a}}$ is paraconsistent. However, this does not mean that we have an argument for $\neg \psi$ in $\Sigma_{\mathrm{c}}$, although there is an argument for $\psi$ in $\Sigma_{\mathrm{c}}$ obviously. Indeed, consider the following counter-example: $\sum=\{(\phi \mathrm{a}),(\neg \phi$ a), $(\neg \phi \vee \psi$ a $)\}$. It is clear that we have an argument for $\psi$ in the knowledge base $\sum$, and $\psi$ is not a free-consequence of $\sum$, but we have no argument which supports $\neg \psi$.

The safely supported consequences can be described in terms of degrees $\operatorname{Cert}(\mathrm{A})$ and Para(A) for arguments A:

Proposition 1: $\Sigma \models$ SS $\psi$ iff there exists an argument A for $\psi$ s.t. $\operatorname{Cert}(\mathrm{A})>\operatorname{Para}(\mathrm{A})$. Proof

Assume $\Sigma \vdash$ SS $\psi$, then let $\mathrm{a}=\operatorname{Cert}(\psi)$ such that $\Sigma_{\mathrm{a}} \vdash$ Free $\psi$. There is an argument $\mathrm{A}$ for $\psi$ in $\operatorname{Free}\left(\Sigma_{\mathrm{a}}\right)$, and $\operatorname{Cert}(\mathrm{A})=\mathrm{a}$. Assume $\operatorname{Para}(\mathrm{A})>\operatorname{Cert}(\mathrm{A})$. Then it means that $\exists\left(\phi, a^{\prime}, b^{\prime}\right) \in \sum^{\prime},\left(\phi, a^{\prime}\right) \in A$, and $b^{\prime}>a$. This means that there is in $\sum$ an argument $\mathrm{B}$ of certainty $\mathrm{b}^{\prime}>\mathrm{a}$ that refutes $\phi$. But this fact contradicts the assumption that $A \subset \operatorname{Free}\left(\Sigma_{\mathrm{a}}\right)$. Conversely suppose $\mathrm{A}$ is an argument for $\psi$ and $\operatorname{Cert}(\mathrm{A})>\operatorname{Para}(\mathrm{A})$. It means that $\mathrm{a}=\operatorname{Cert}(\mathrm{A})>\max \left\{\mathrm{b}_{\mathrm{i}},\left(\phi, \mathrm{a}_{\mathrm{i}}\right) \in \mathrm{A},\left(\phi, \mathrm{a}_{\mathrm{i}}, \mathrm{b}_{\mathrm{i}}\right) \in \Sigma^{\prime}\right\}$. Hence if $\mathrm{B}$ is an argument for $\neg \phi, \mathrm{B}$ is not a subset of $\sum_{\mathrm{a}}$, and $\phi$ is free in $\Sigma_{\mathrm{a}}$. Hence $\operatorname{A\subseteq Free}\left(\sum_{\mathrm{a}}\right)$, and $\sum_{\mathrm{a}} \vdash$ Free $\psi$.

Moreover the set of safely supported consequences of $\sum$ is consistent:

Proposition 2: Let $\mathrm{K}=\left\{\psi \mid \sum \vdash \mathrm{SS} \psi\right\}$. Then the set of formulas $\mathrm{K}$ is consistent.

Lemma: The set of formulas $\bigcup_{\mathrm{i}=1, \mathrm{n}} \operatorname{Free}\left(\Sigma_{\mathrm{a}_{\mathrm{i}}}\right)$ is consistent.

Proof

Free $\left(\sum_{\mathrm{a}_{\mathrm{n}}}\right)=\operatorname{Free}\left(\sum\right)$ is consistent. Then Free $\left(\sum_{\mathrm{a}_{\mathrm{n}-1}}\right) \cup \operatorname{Free}\left(\sum_{\mathrm{a}_{\mathrm{n}}}\right)$ is also consistent. Indeed assume it is not the case, it means that $\exists \mathrm{A} \subseteq \operatorname{Free}\left(\Sigma_{\mathrm{a}_{\mathrm{n}-1}}\right)$, $\mathrm{A} \cup \operatorname{Free}\left(\sum_{\mathrm{a}_{\mathrm{n}}}\right)-\perp$ where both $A$ and Free $\left(\Sigma_{a_{n}}\right)$ are consistent. This contradicts the fact that Free $\left(\Sigma_{a_{n}}\right)$ only contains free formulas in $\sum_{a_{n}}$ (since $A \subset \Sigma_{a_{n}}$ ). More generally, assume $\operatorname{Free}\left(\Sigma_{\mathrm{a}_{\mathrm{i}}}\right) \cup \ldots \cup \operatorname{Free}\left(\Sigma_{\mathrm{a}_{\mathrm{n}}}\right)=\mathrm{F}$ is consistent; let $\mathrm{A} \subseteq$ Free $\left(\sum_{\mathrm{a}_{\mathrm{i}-1}}\right)$, and assume $A \cup F \vdash \perp$. Let $j$ be the smallest rank such that $A \cup$ Free $\left(\Sigma_{a_{i}}\right) \cup \ldots \cup \operatorname{Free}\left(\Sigma_{a_{j}}\right)$ is inconsistent. This contradicts the fact that $\operatorname{Free}\left(\Sigma_{\mathrm{a}_{\mathrm{j}}}\right)$ only contains free formulas in $\Sigma_{\mathrm{a}_{\mathrm{j}}}$ (since $\mathrm{A} \subseteq \Sigma_{\mathrm{a}_{\mathrm{j}}}$ as well as $\operatorname{Free}\left(\Sigma_{\mathrm{a}_{\mathrm{i}}}\right) \subseteq \sum_{\mathrm{a}_{\mathrm{j}}}$ for $\left.\mathrm{i} \leq \mathrm{j}\right)$.

Proof of Proposition 2

We use the previous lemma. Clearly for each $\phi$ in $\mathrm{K}$, there exists an argument say $\mathrm{A}(\phi)$, in $\bigcup_{\mathrm{i}=1, \mathrm{n}} \operatorname{Free}\left(\sum_{\mathrm{a}_{\mathrm{i}}}\right)$. Therefore $\bigcup_{\phi \in \mathrm{K}} \mathrm{A}(\phi) \subseteq \bigcup_{\mathrm{i}=1, \mathrm{n}} \operatorname{Free}\left(\sum_{\mathrm{a}_{\mathrm{n}}}\right)$ and hence $\bigcup_{\phi \in \mathrm{K}} \mathrm{A}(\phi)$ is consistent as well as its deductive closure.

Notice that when the knowledge base is flat (i.e., without any priority between their elements) the safely supported consequence relation as well as CP-consequence and $\mathrm{PC}$-consequence relations are equivalent to the free-consequence relation defined in Section 2. Moreover, we can show that even if $\beta$ and $\delta$ are safely supported consequences of $\sum$, their conjunction is not necessarily a safely supported consequence of $\sum$. Indeed, let us consider the following counter-example where our knowledge base is $\Sigma=\{(\alpha 1),(\neg \alpha \vee \beta .9),(\neg \rho \vee \delta .8),(\rho .7),(\neg \alpha .7)\}$. It is clear that $\beta$ and $\delta$ are both safely supported consequences of $\sum$ (since $\sum .9-$ Free $\beta$ and $\sum .7-$ Free $\delta$ ), while $\beta \wedge \delta$ is not since there is no $a>0$ such that $\Sigma_{\mathrm{a}}-$ Free $\beta \wedge \delta$. Indeed, a part of the argument 
$\{(\neg \rho \vee \delta .8),(\rho .7)\}$ for $\delta$ (namely $\rho)$ has a certainty not greater than the level of paraconsistency of a part of the argument for $\beta$ (namely $\alpha$ ). This remark also holds for PC-consequence and CP-consequence (we can use the same counter-example). The failure of the "AND" property should not be a surprise when dealing with multi-source inconsistent information.

\section{Comparative study and discussion}

We first start this section by comparing the three above-mentioned inconsistencytolerant consequence relations. To this aim we use the following example where the knowledge base is: $\Sigma=\{(\phi \mathrm{a}),(\neg \phi \mathrm{b}),(\neg \phi \vee \psi \mathrm{c}),(\chi \mathrm{d}),(\neg \chi \vee \psi \mathrm{e}),(\neg \chi \mathrm{f})\}$.

Then: $\Sigma^{\prime}=\{(\phi \mathrm{a} \mathrm{b}),(\neg \phi \mathrm{b}$ a $),(\neg \phi \vee \psi \max (\mathrm{b}, \mathrm{c}) 0),(\chi \mathrm{d} \mathrm{f}),(\neg \chi \vee \psi \max (\mathrm{e}, \mathrm{f}) 0),(\neg \chi \mathrm{fd})\}$.

Notice that only the formulas $\neg \phi \vee \psi$ and $\neg \chi \vee \psi$ are free in $\sum$, all the others are paraconsistent. We are interested in knowing if $\psi$ can be deduced from $\sum$. We have:

$\operatorname{Label}(\psi)=\{(\min (\mathrm{a}, \mathrm{c}) \mathrm{b}),(\min (\mathrm{d}, \mathrm{e}) \mathrm{f}))\}$

obtained using the following arguments respectively: $\mathrm{A}=\{(\phi \mathrm{a}),(\neg \phi \vee \psi \mathrm{c})\}$, and $\mathrm{B}=$ $\{(\chi \mathrm{d}),(\neg \chi \vee \psi \mathrm{e})\}$. Then:

- Assume that $\mathrm{a}>\mathrm{c}>\mathrm{b}>\mathrm{f}>\mathrm{d}>\mathrm{e}$. Then minimizing the paraconsistency degree of the conclusion $\psi$ will lead to select $\mathrm{B}$ as the best argument for $\psi$, and since $\min (\mathrm{d}, \mathrm{e})<\mathrm{f}$ then $\psi$ will not be PC-inferred. This result is somewhat debatable since in the argument $\mathrm{A}, \psi$ is inferred from the two most certain formulas in $\sum$. In contrast, $\mathrm{A}$ is the strongest argument for $\psi$ and hence $\psi$ is a CP-consequence of $\sum$ (since $\min (a, \max (b, c))>b)$. More generally, if we have a pair $(x y)$ with $y>x$ and $y$ is the lowest paraconsistency degree in $\operatorname{Label}(\psi)$ then $\psi$ is completely inhibited by PCconsequence even if we have $(1 \mathrm{z})$ in $\operatorname{Label}(\psi)$ with $\mathrm{z}$ slightly greater than $\mathrm{y}$. Notice from the example, that the $\mathrm{PC}$-consequence relation does not recover all the possibilistic consequences of the knowledge base (e.g., here $\psi$ is a possibilistic consequence of the knowledge base). In contrast, $\mathrm{CP}$-consequences do recover all the possibilistic consequences of the knowledge base.

- Assume that $\mathrm{a}>\mathrm{b}>\mathrm{c}>\mathrm{d}>\mathrm{e}>\mathrm{f}$ then we obtain $\psi$ as a plausible consequence of $\sum$ if we first minimize the paraconsistency degree of $\psi$. In contrast, if we first maximize the certainty degree, $\mathrm{A}$ is selected since $\mathrm{A}$ is the best argument of $\psi$ in this case, but $\psi$ is no longer inferred since $b>\min (a, \max (c, b))$. This result is somewhat debatable especially if the certainty degree of $\neg \chi$ is very low. And more generally, if we have a pair ( $\mathrm{x} y$ ) with $\mathrm{y}>\mathrm{x}$ and $\mathrm{x}$ is the highest certainty degree in $\operatorname{Label}(\psi)$ then $\psi$ is completely inhibited by CP-consequence even if we have $(\mathrm{z} 0)$ in $\operatorname{Label}(\psi)$ (namely a free proof for $\psi$ with $\mathrm{z}<\mathrm{x}$ ).

- Notice that in the two above cases, $\psi$ is a safely supported consequence of $\Sigma$ when we apply the third approach. Indeed, $\Sigma_{\mathrm{c}}=\{(\phi \mathrm{a}),(\neg \phi \vee \psi \mathrm{c})\} \vdash$ Free $\psi$ in the first case, and $\sum_{\mathrm{e}}=\{(\phi \mathrm{a}),(\neg \phi \mathrm{b}),(\neg \phi \vee \psi \mathrm{c}),(\chi \mathrm{d}),(\neg \chi \vee \psi \mathrm{e})\} \vdash$ Free $\psi$ in the second case. Indeed $\operatorname{Cert}(\mathrm{A})>\operatorname{Para}(\mathrm{A})$ in the first case and $\operatorname{Cert}(\mathrm{B})>\operatorname{Para}(\mathrm{B})$ in the second case. The following proposition generalizes this remark and shows that $\vdash \mathrm{SS}$ produces more results than $\vdash \mathrm{PC}$ and $\vdash \mathrm{CP}$.

Proposition 3: If $\Sigma-\mathrm{PC} \psi($ resp. $\Sigma \models \mathrm{CP} \psi)$ then $\Sigma \models$ SS $\psi$.

Proof

Indeed, if $\psi$ is a PC-consequence (or a CP-consequence) of $\sum$ then there exists an argument $\mathrm{A}$ for $\psi$ in $\sum$ such that $\mathrm{a}=\mathrm{Cert}(\mathrm{A})>\operatorname{Para}(\mathrm{A})$. Then use Proposition 1 .

Furthermore, in [2] we have shown that - SS recovers all the possibilistic consequences of $\sum$. Using Proposition 1 we can show that if there is in $\operatorname{Label}(\psi)$ a 
pair (a b) with a $>$ b then $\Sigma-$ SS $\psi$. This means that it cannot be that both $\psi$ and $\neg \psi$ are $\mathrm{PC}$-consequence or $\mathrm{CP}$-consequence of $\Sigma$. Indeed, assume that both $\psi$ and $\neg \psi$ are PC-consequences of $\sum$, namely there is a pair $(\mathrm{a} b) \in \operatorname{Label}(\psi)$ with $\mathrm{a}>\mathrm{b}$, and a pair (c d) $\in \operatorname{Label}(\neg \psi)$ with $\mathrm{c}>\mathrm{d}$, therefore using the remark above both $\psi$ and $\neg \psi$ are paraconsistent consequences of $\Sigma$, and this is not possible using Proposition 2. Moreover, as suggested by the previous examples, we may have $(a \mathrm{~b}) \in \operatorname{Label}(\psi)$ with $\mathrm{a}>\mathrm{b}$ (in this case $\psi$ is a safely supported consequence), while $\psi$ is neither a CPconsequence nor a PC-consequence of the knowledge base. Indeed, it is sufficient to imagine a situation where $\operatorname{Label}(\psi)=\left\{(\mathrm{a}+\varepsilon, 1),(\mathrm{a}, \mathrm{b}),\left(\varepsilon^{\prime}, \mathrm{b}-\varepsilon^{\prime \prime}\right)\right\}$ with $1>\mathrm{a}>\mathrm{b}>\mathrm{b}-\varepsilon^{\prime \prime} \geq \varepsilon^{\prime}$.

\section{Conclusion}

This paper has investigated a local approach to deal with inconsistency. Three consequence relations have been proposed and all are safe, namely we cannot have inconsistent sets of consequences of the knowledge base. Moreover, we have shown that only two of the consequence relations (CP-consequence and safely supported consequence relations) recover all the possibilistic results. On the other hand, the safely supported consequence relation generates more results than the $\mathrm{CP}$-consequence, and hence - SS seems to be a better approach to deal with inconsistency in a local way. Besides, in [2] we have shown that the so-called preferred sub-theories approach [4] generates more results than the safely supported consequence relation. However, some results given by preferred sub-theories may be debatable in a multi-source inconsistency reasoning perspective, as we can see in the following example: $\sum=\{(\phi$ 1.), ( $\neg \phi .9),(\neg \phi \vee \psi .8)\}$. Here $\psi$ is a plausible consequence of $\sum$ using the preferred sub-theories approach (since we remove simply $\neg \phi$ from the knowledge base) although the paraconsistency degree of $\psi$ is greater than its certainty. The consequence relation $-\mathrm{SS}$ which is based on undefeated arguments (since the inference requires the existence of a "free" argument) seems to be more satisfactory for reasoning with (possibly inconsistent) information coming from different sources. Lastly in [2] it is established that if the set of safely supported consequences of $\sum$ is completed by deductive closure, then the obtained closed set is the set of possibilistic consequences of the intersection of all preferred sub-bases in the sense of Brewka [4].

\section{References}

1. S.Benferhat, D.Dubois, H.Prade. Representing default rules in possibilistic logic. Proc. KR'92, Cambridge, MA, Oct 26-29, 1992, pp. 673-684.

2. S.Benferhat, D.Dubois, H.Prade. Some syntactic approaches to the handling of inconsistent knowledge bases. Tech. Report IRIT/94-55-R, Univ. P.Sabatier, Toulouse.

3. P.Besnard, J.Lang. Possibility and necessity functions over non-classical logics. Proc. UAI'94, Morgan Kaufmann, pp. 69-76.

4. G.Brewka. Preferred subtheories: an extended logical framework for default reasoning. Proc. IJCAI'89, Detroit, MI, August, 1989, pp. 1043-1048.

5. D.Dubois, J.Lang, H.Prade. Handling uncertainty, context, vague predicates, and partial inconsistency in possibilistic logic. In: Fuzzy Logic and Fuzzy Control (Proc. IJCAI'91 Workshops) (D.Driankov et al., eds), Springer Verlag, LNAI N833, pp. 4555, 1994.

6. D.Dubois, J.Lang, H.Prade. Possibilistic logic. In: Handbook of Logic in Artificial Intelligence and Logic Programming, Vol. 3 (D.M.Gabbay et al., eds.), Oxford University Press, pp. 439-513, 1994.

7. N.Rescher, R. Manor. On inference from inconsistent premises. Theory and Decision, 1, 179-219, 1970 .

8. G.R.Simari, R.P.Loui. A mathematical treatment of defeasible reasoning and its implementation. Artificial Intelligence, 53, 125-157, 1992. 